\title{
CIÊNCIAS AMBIENTAIS
}

\section{GEOPROCESSAMENTO NA ANÁLISE ESPAÇO-TEMPORAL DA COBERTURA VEGETAL DO MUNICÍPIO DE SERIDÓ - PB}

\author{
DOI: http://dx.doi.org/10.31072/rcf.v9i1.540 \\ GEOPROCESSING IN THE SPATIAL-TEMPORAL ANALYSIS OF THE VEGETATION \\ COVER OF THE CITY OF SERIDÓ - PB
}

Sabrina Cordeiro de Lima1; Felipe Cordeiro de Lima²; Rafaela Felix Basílio³; João Miguel de Moraes Neto ${ }^{4}$.

\begin{abstract}
RESUMO: A utilização das técnicas de geoprocessamento e de sensoriamento remoto nos estudos de quantificação da cobertura vegetal contribui para a eficiência e confiabilidade nas análises que envolvem os processos de degradação da vegetação natural, já que os índices de vegetação são parâmetros indicadores de qualidade ambiental. O Seridó Oriental paraibano, é uma região que apresenta fortes indícios de degradação ambiental devido ao uso e exploração inadequados dos seus recursos naturais. Este estudo teve como objetivo analisar a dinâmica espaço-temporal da cobertura vegetal do solo no município de Seridó PB, nos anos de 1990 e 2013. Foram elaborados mapas de Índices de Vegetação por Diferença Normalizada (IVDN) e as Composições Multiespectrais Ajustadas (CMA) por intermédio do software SPRING, versão 5.4.2, utilizando imagens orbitais TM/LANDSAT 5 para o ano de 1990 e TM/LANDSAT 8 para o ano de 2013 adquiridas no acervo de imagens do Instituto Nacional de Pesquisas Espaciais (INPE). A escala temporal de análise permitiu verificar ao longo do período de 23 anos a variação da cobertura vegetal da região, caracterizada pelo aumento das áreas degradadas devido a atividades antrópicas, tornando a região imprópria para as atividades agrícolas.
\end{abstract}

Palavras-chave: Índices de Vegetação por Diferença Normalizada. IVDN. Composições multiespectrais ajustadas. Degradação.

\begin{abstract}
The use of geoprocessing and remote sensing techniques in the studies of quantification of the vegetation cover contributes to an efficiency and reliability in the analyzes that involve the processes of degradation of the natural vegetation, since the vegetation indexes are indicators of environmental quality. The Eastern Seridó Paraíba is a region that presents strong indications of environmental degradation due to the inadequate use and exploitation of natural resources. This study aimed to analyze the spatial-temporal
\end{abstract}

\footnotetext{
${ }^{1}$ Engenheira Agrícola, Mestranda em Engenharia Agrícola, Universidade Federal de Campina Grande. ORCID: https://orcid.org/0000-0002-9597-2317;

2 Prof. Mestre, graduado em Engenharia Ambiental, docente da Faculdade de Educação e Meio Ambiente Ariquemes -RO. ORCID: https://orcid.org/0000-0002-7345-2011;

${ }^{3}$ Engenheira Agrícola, Mestranda em Engenharia Agrícola, Universidade Federal de Campina Grande. ORCID: https://orcid.org/0000-0002-7638-2813;

4 Doutor, docente da Universidade Federal de Campina Grande - Campina Grande - PB. ORCID: https://orcid.org/0000-0001-7672-5540.
} 
dynamics of vegetal soil cover of Seridó city in Paraiba, in the years 1990 and 2013. Have been elaborated Normalized Difference Vegetation Index (NDVI) maps and Adjusted multispectral compositions (AMC) Using SPRING software, version 5.4.2, using orbital images TM / LANDSAT 5 for the year 1990 and TM / LANDSAT 8 for the year 2013 acquired in the collection of images of the Instituto Nacional de Pesquisas Espaciais (INPE). The temporal scale of analysis allowed to verify throughout the period of 23 years the variation of the vegetation cover of the region, characterized by the increase of degraded areas due to anthropic activities, rendering the region unfit for agricultural activities.

Keywords: Vegetation Indexes by Normalized Difference.NDVI. Adjusted multispectral compositions. Degradation.

\section{INTRODUÇÃO}

Inserido no Bioma Caatinga, no semiárido paraibano, o município de Seridó vem sofrendo alterações em sua vegetação. Segundo Silva ${ }^{(1)}$, a preservação da cobertura vegetal é a condição básica para a conservação dos recursos hídricos, visto que a vegetação tem importante função em proteger e manter as nascentes e cursos d'água.

Pesquisas indicando a variação do ambiente e os impactos causados por tais mudanças vêm sendo desenvolvidas para o semiárido nordestino, região esta, com alta vulnerabilidade a mudanças ambientais devido aos baixos índices de pluviosidade e que enfrentam um amplo número de questões relacionadas com o manejo de seus recursos naturais. A região do Seridó caracteriza-se por estar em processo de desertificação, o que segundo Alves(2), é decorrente da utilização inadequada dos recursos naturais, de técnicas agrícolas inapropriadas e, especialmente de modelos de desenvolvimento econômicos de curto prazo.

As tecnologias de geoprocessamento e de sensoriamento remoto são fundamentais para monitoramento dos recursos naturais. Investimentos expressivos têm sido feitos para o desenvolvimento e 0 uso dessas tecnologias, principalmente no Instituto Nacional de Pesquisas Espaciais (INPE) (3). O software Sistema de Processamento de Informações Georrefenciadas (SPRING) é um Sistema de Informação Geográfica (SIG) disponibilizado pelo Instituto Nacional de Pesquisas Espaciais (INPE). Os dados de sensoriamento remoto têm sido fundamentais em pesquisas direcionadas ao estudo do comportamento dinâmico da vegetação ${ }^{(4)}$. As informações produzidas com os dados dos sensores do satélite são de grande importância para o estudo espaçotemporal do ambiente geográfico podendo 
identificar a degradação das áreas e os índices de vegetação.

Com 0 objetivo de analisar quantitativamente e qualitativamente a situação da vegetação a partir de imagens de satélites, foram desenvolvidos os índices de vegetação com a finalidade de reduzir o número de parâmetros presentes nas medidas multiespectrais ${ }^{(5)}$. $O$ índice vegetativo mais utilizado é conhecido atualmente como índice de vegetação da diferença normalizada (IVDN). Dessa forma, tem-se o monitoramento da densidade e da situação de vigor da vegetação verde sobre a superfície terrestre (6). O IVDN é uma variável expressivamente afetada pelo regime de precipitação pluviométrica da região analisada (7).

As composições coloridas para mapear as áreas vegetadas permitem a observação mais facilitada do desenvolvimento da vegetação em cada banda associada a uma das cores primárias (azul, verde e vermelho), no que Ihe concerne, agrupadas em suas variadas composições, além de serem eficientes e práticas no estudo e acompanhamento espaço-temporal da ação antrópica ${ }^{(8)}$.

Com objetivo de mapear a alteração nas classes de cobertura vegetal, solo e água no município de Seridó-PB, no período 1990 e 2013 desenvolveu-se esta pesquisa, utilizando-se dos índice de vegetação por diferença normalizada (NDVI) e das composições multiespectrais ajustadas (CMA) como parâmetros.

\section{MATERIAIS E MÉTODOS}

O município de Seridó (Figura 1) está localizado no Seridó Oriental do estado da Paraíba a uma latitude $06^{\circ} 56^{\prime} 03^{\prime \prime}$ sul e a uma longitude $36^{\circ} 24^{\prime} 08^{\prime \prime}$ oeste, e a uma altitude de 631 metros. Possui uma área de $227,7 \mathrm{~m}^{2}$ e um distrito, São Vicente do Seridó.

O clima da área de estudo é caracterizado do tipo Bsh - Semiárido quente, de acordo com a classificação de Köppen. No município a pluviosidade média anual é de cerca de $500 \mathrm{~mm}$, sendo o período chuvoso concentrado entre os meses de fevereiro e maio, com forte variação ao longo do tempo e do espaço. A vegetação predomina a caatinga hiperxerófila. As temperaturas médias são de $27^{\circ} \mathrm{C}$, o que ocasiona o déficit hídrico presente na maioria dos meses.

Figura 1 - Localização geográfica do município de Seridó - PB.

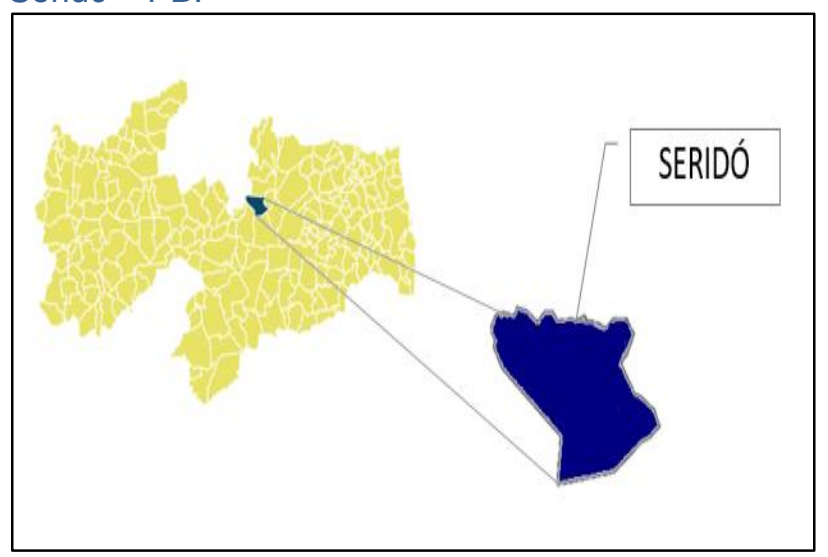


Para observação espaço-temporal da cobertura vegetal na região de estudo foram elaborados mapas, a partir das imagens orbitais de satélite especificadas na Tabela 1, que foram cedidas pela divisão de geração de imagens (DGI), do
Instituto Nacional de Pesquisas Espaciais (INPE), com resolução espacial de $30 \mathrm{~m}$, sendo estas analisadas pelo software SPRING ${ }^{(3)}$ versão 5.4.2.

Tabela 1 - Identificação das imagens orbitais com cobertura para a área de estudo.

\begin{tabular}{ccccc}
\hline Satélite & Sensor & Órbita & Ponto & Data \\
\hline LANDSAT 5 & TM & 215 & 65 & $14 / 03 / 1990$ \\
LANDSAT 8 & TM & 215 & 65 & $04 / 08 / 2013$ \\
\hline
\end{tabular}

A escolha dos satélites LANDSAT $5 \mathrm{e}$ LANDSAT 8 para a execução do trabalho, considerou a resolução espectral satisfatória das imagens e a disponibilidade na data de passagem atualizada no acervo de imagens de satélite do Instituto Nacional de Pesquisas Espaciais (INPE). Através das imagens, foi então levantada a situação da cobertura vegetal para dois períodos distintos, 1990 e 2013. Realizou-se, durante 23 anos, um estudo comparativo entre as propriedades espectrais dos alvos da superfície terrestre (Vegetação, Solo e Água).

$\mathrm{Na}$ quantificação da cobertura vegetal utilizou-se como plano de informações as composições multiespectrais ajustadas (RGB) nas bandas 1 a 3 , e IVDN nas bandas 3 e 4 .

No processamento digital das imagens utilizou-se a técnica de realce e contrastes que tem por objetivo aprimorar a qualidade das imagens sob critérios subjetivos do olho humano ${ }^{(9)}$. Esta técnica visa equalizar as bandas (10). Para a correção dos níveis de cinza da composição colorida, utilizou-se o ajuste linear. Essas técnicas são normalmente utilizadas como uma fase de préprocessamento para sistemas de reconhecimento de padrões (8). Estas imagens são realçadas, expandindo o intervalo de intensidade e saturação por meio das técnicas convencionais de contraste, e voltam a serem transformadas para o sistema RGB, permitindo, dessa forma, a melhor separação das feições na qual deseja-se observar ${ }^{(11)}$.

Com o objetivo de ampliar o contraste entre vegetação e solo, empregou-se as operações aritméticas (razão entre bandas) associados ao 
vermelho e infravermelho próximo, compondo assim, os chamados índices de vegetação por diferença normalizada (IVDN) (Eq. 1).

$$
\mathrm{C}=\mathrm{G} * \frac{(\mathrm{A}-\mathrm{B})}{\mathrm{A}+\mathrm{B}}+\mathrm{O}(\mathrm{Eq} \cdot 1)
$$

Onde, $A=$ banda infravermelho próximo/banda $4 ; \mathrm{B}=$ banda vermelho / banda 3; $\mathrm{G}=$ ganho (256) e $\mathrm{O}=$ offset (64).

As Composições multiespectrais ajustadas (b3 + IVDN + b1) correspondem a uma transformação RGB onde a fonte de luz vermelha $(\mathrm{R})$ está posicionada na banda 3 , na fonte verde $(G)$ a imagem IVDN e na fonte azul (B) a banda 1. Nesta combinação, as áreas com alto valor de
IVDN, aparecerão em verde (ocorrência de vegetação), e as áreas de IVDN de baixa ocorrência aparecerão em magenta, resultante da adição do vermelho com azul, o que indica a presença de solos expostos (12).

\section{RESULTADOS E DISCUSSÕES}

Após o processamento das imagens orbitais, confeccionaram-se os mapas básicos e a formação de resultados com o índice de vegetação por diferença normalizada (IVDN), nas imagens de 1990 e 2013 (Figuras 2 e 3).

Figura 2 - IVDN do ano 1990 (Imagem TM/LANSAT 5 de 14/03/1990 órbita 215/65).

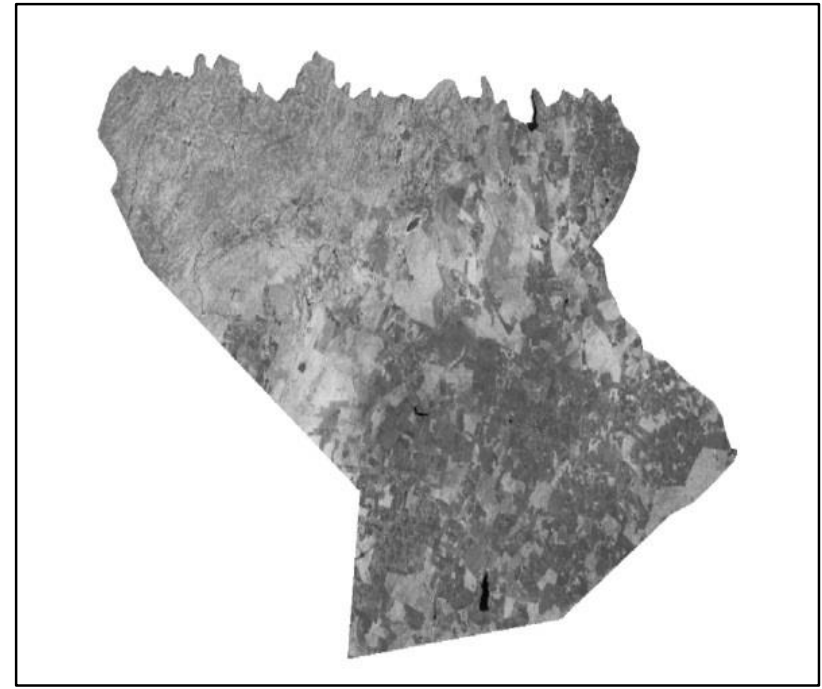


Figura 3 - IVDN do ano 2013 (Imagem

TM/LANSAT 8 de 04/08/2013 órbita 215/65).

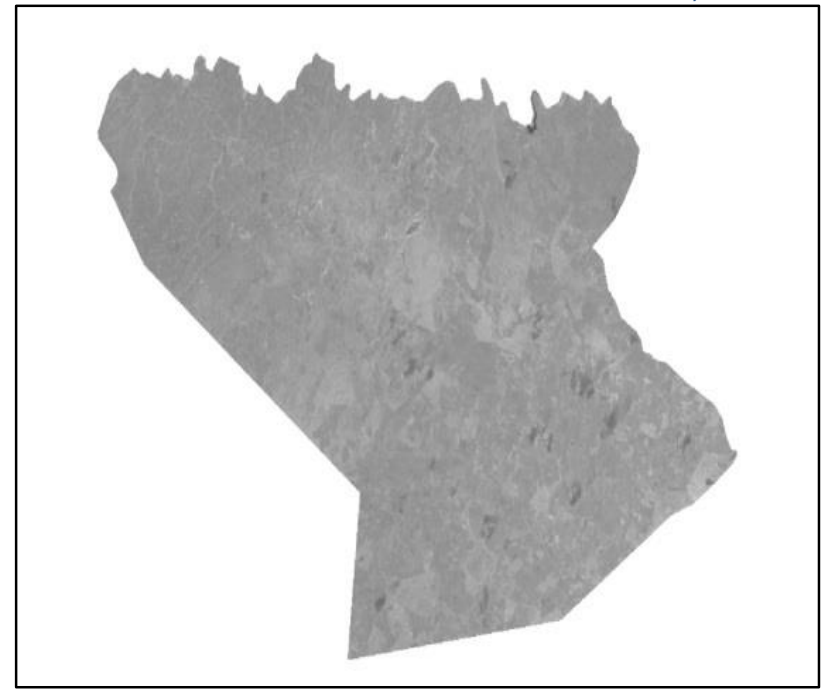

Observando o comportamento das imagens IVDN mostraram-se diferentes com relação a cobertura vegetal da região. A paisagem mostra um processo de perda da vegetação, onde a área representada por tons de cinza mais escuros tendem a ser de solo exposto e a mais clara à cobertura vegetal (Figuras 2 e 3 ).
A análise comparativa das Composições Multiespectrais Ajustadas (CMA) para os anos de 1990 e 2013, onde permitiu analisar o comportamento dos alvos, vegetação, solo e água encontramse nas Figuras 4 e 5.

Figura 4 - CMA do ano 1990 (Imagem TM/ LANSAT 5 de 14/03/1990 órbita 215/65).

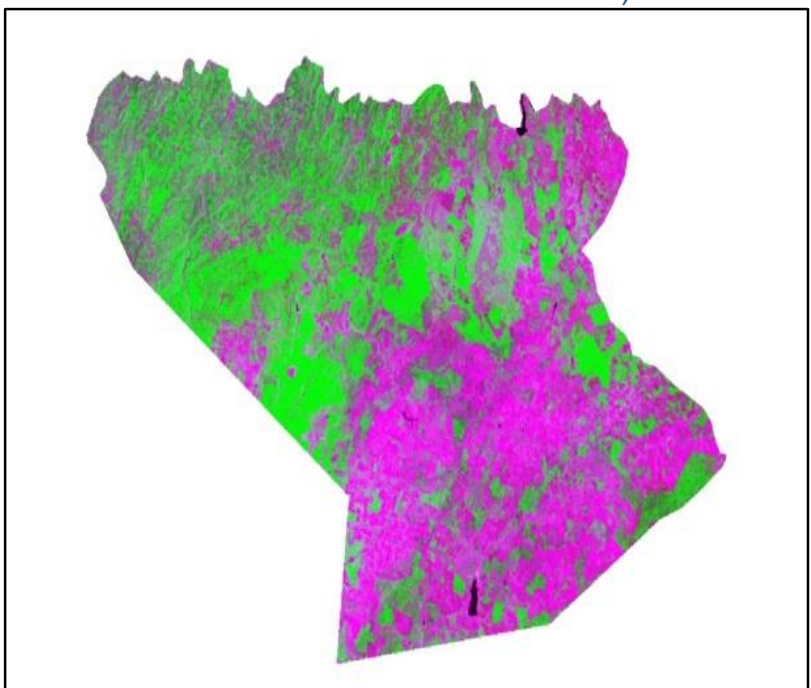


Figura 5 - CMA do ano 2013 (Imagem TM/

LANSAT 8 de 04/08/2013 órbita 215/65.

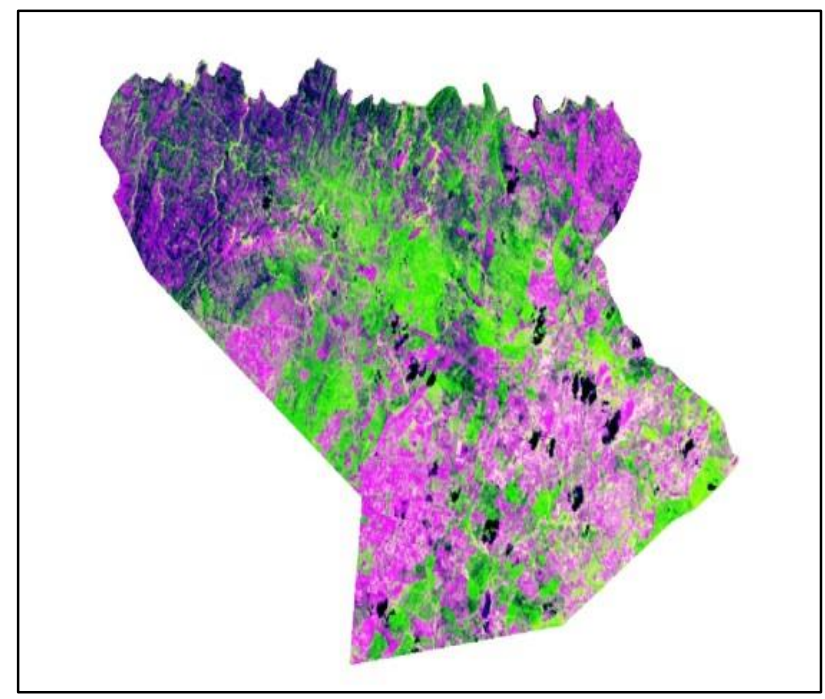

Nas Figuras 4 e 5 observa-se na cor verde as áreas com cobertura vegetal, enquanto as áreas de solo exposto ou com cobertura vegetal rala são representadas pelas cores magenta, o que torna evidente a intermitente ação homem no ambiente natural.

Observa-se que este município apresentava, tanto para o ano de 1990 como para 2013, uma cobertura vegetal já prejudicada, comprovada pela extensão das áreas de solo exposto. As figuras expressam uma devastação, resultante da ação antrópica na alteração dos modelos de organização social e econômica da região, comprometendo, por consequência, 0 processo de desenvolvimento, considerando-se 0 expressivo aumento da população e consequentemente $\mathrm{o}$ aumento da área urbana.
Verifica-se na Figura 4 para o 1990 que a coloração magenta é mais vibrante dando a entender a ocorrência de áreas mais degradadas, com destaque à sul e nordeste da região de estudo. A região noroeste do município apresenta um aumento da degradação ambiental no ano de 2013 (Figura 5). Os corpos d'água cuja tonalidade é azul escuro, com seus limites e contornos bem definidos nas imagens sintéticas apresentam pouca variação (Figura 4 e 5).

As áreas com maiores altitudes possivelmente dificultam a utilização das suas terras para plantio dos cultivos mais exercidos na região, o que confere a conservação da vegetação existente nessa área ${ }^{(13)}$.

O uso e ocupação das terras nos municípios do Cariri, a exemplo do que predomina nessa parte do Brasil, 
fundamentam-se historicamente na agricultura (principalmente de subsistência), extrativismo vegetal (produção de lenha e carvão vegetal) e pecuária extensiva (particularmente caprina), em propriedades que vem reduzindo de tamanho ao longo da história, devido as consecutivas divisões por questões de herança familiar (14).

O cariri ocidental paraibano é uma região com sua economia baseada na extração mineral, em Seridó predomina-se a exploração de Feldspato e Quartzo. A mineração é uma atividade que causa alta degradação no solo.

A consequente exposição direta do solo à ação da erosão impossibilita na vegetação desta área uma mudança em termos qualitativos e quantitativos, associada às condições climáticas desfavoráveis ${ }^{(15)}$.

Segundo Souza ${ }^{(16)}$, esse conjunto de fatores levam os municípios da caatinga que já foram estudados a terem seus territórios atingidos pela desertificação, apesar de algumas áreas apresentarem bons níveis de preservação da Caatinga e uso sustentável dos solos.

No caso do município de Seridó PB, os solos são classificados como
Neossolos Litólicos Eutróficos e Neossolos Regolíticos, com terras não cultivadas com severas limitações para culturas permanentes e reflorestamento; além de serem íngremes e mais susceptíveis a erosão, próprias para cultivos contínuos e que se prestam mais para lavoura esporádica (17).

\section{CONCLUSÃO}

A escala temporal de análise $(1990$ e 2013) permitiu verificar ao longo do período de 23 anos a variação da cobertura vegetal da região, caracterizada pelo aumento das áreas degradadas devido a atividades antrópicas. É necessário chamar a atenção para o impacto ambiental causado pela descaracterização da cobertura vegetal natural, que foi apresentado na região de estudo, caracterizando indícios da desertificação.

O monitoramento da variação espaço-temporal da vegetação natural de uma região utilizando imagens de satélite podem ser executados com eficácia pelo software SPRING, através das imagens IVDN e Composições Multiespectrais Ajustadas (CMA). 


\section{REFERÊNCIAS}

1. Silva RMP, Lima JR, De Mendonça IFC. Alteração da cobertura vegetal na SubBacia do Rio Espinharas de 2000 a 2010. Revista Brasileira de Engenharia Agrícola e Ambiental-Agriambi 2014; 18(2): 202-209.

2. Alves JJA, Do Nascimento SS, De Souza EN. Núcleos de desertificação no Estado da Paraíba. RA'E GA-O Espaço Geográfico em Análise 2009; 17: 139-152.

3. Câmara G, Souza RCM., Freitas UM, \& Garrido J. SPRING: Integrating remote sensing and GIS by object-oriented data modelling. Computers \& graphics, 1996; 20(3): 395-403.

4. Dantas FDC, Braga CC, Souza EP, Silva STA. Determinação do albedo da superfície a partir de dados AVHRR/NOAA e TM/LANDSAT-5. Revista Brasileira de Meteorologia, São Paulo, 2010; 25(1): 2431.

5. Francisco PRM, de Brito Chaves I, Chaves LHG, de Lima ERV. Detecção de Mudança da Vegetação de Caatinga (Change Detection of Vegetation Caatinga). Revista Brasileira de Geografia Física 2013; 5(6): 1473-1487.

6. Francisco PRM, de Brito Chaves I, Chaves LHG, de Lima ERV, da Silva BB. Análise espectral e avaliação de índices de vegetação para 0 mapeamento da caatinga. Revista Verde de Agroecologia e Desenvolvimento Sustentável 2015; 10(3): 01-12.

7. Aquino CMS, Oliveira JGB. Estudo da dinâmica do Índice De Vegetacão Por Diferença Normalizada (NDVI) no núcleo de São Raimundo Nonato-PI. GEOUSP: Espaço e Tempo (Online) 2012; (31): 157168.

8. Silva Neto AF et al. As composições multiespectrais ajustadas e a dinâmica vegetal em municípios do Cariri Ocidental da Paraíba - Brasil. Anais XIV Simpósio Brasileiro de Sensoriamento Remoto. 2530 abril; Natal-RN; Brasil; p.6249-6255; 2009.

9. Manual do SPRING: Noções de Geoprocessamento. São José dos Campos (SP): Instituto Nacional de Pesquisas Espaciais; 1997.

10. Coelho IDAM, Duarte SMA, Coelho ODAM. Estudo da dinâmica da cobertura vegetal no município de Floresta/PE através de processamento digital de imagens. Revista Geama 2016; 1(2): 187204.

11. Rios RO, Almeida MG, Ribeiro MM. Análise do uso e ocupação do solo da cidade de Vitória-ES usando a composição colorida de imagens fornecidas pelo satélite landsat-5. XIII Encontro de modelagem Computacional Instituto Politécnico (IPRJ), UERJ, Nova Friburgo (RJ): Brasil: p. 03-05, 2010.

12. de Farias AA, Souza JTA, Alves TLB, de Alcântara Silva VM, Neto JMM. Degradação Ambiental no Entorno da Bacia Hidráulica do Açude Manoel Marcionilo, Taperoá-PB. Revista Brasileira de Geografia Física 2012; 5(4): 863-876.

13. Silva GJF, Almeida NV. Degradação ambiental no município de Parari-PB: uma análise por meio de sensoriamento remoto. Revista Geografar 2016; 10(2): p. 140-164.

14. Souza BI, Menezes, R, Cámara Artigas R. Efeitos da desertificação na composição de espécies do bioma Caatinga, Paraíba/Brasil. Investigaciones geográficas 2015; (88); 45-59.

15. Costa TC, de Oliveira MA, Accioly LJDO, da Silva FH. Análise da degradação da caatinga no núcleo de desertificação do Seridó (RN/PB). Revista Brasileira de 
Engenharia Agrícola e Ambiental 2009; 13(Suplemento); p.961-974.

16. Souza BI. Uso da vegetação e solos em áreas susceptíveis à desertificação na Paraíba/Brasil. GEOgraphia 2012; 13(25); 77-105.
17. AESA - Agência Executiva de Gestão das Águas do Estado da Paraíba. SIGaesa-web. Mapa interativo, 2010.

\section{Como citar (Vancouver)}

Lima SC, Lima FC, Basílio RF, Moraes Neto JM. Geoprocessamento na análise espaço-temporal da cobertura vegetal do município de Seridó - PB. Rev Cient Fac Educ e Meio Ambiente [Internet]. 2018;9(1):266-275. DOI: http://dx.doi.org/10.31072/rcf.v9i1.540 